\title{
Grapes Leaf Disease Detection using Convolutional Neural Network
}

\author{
Tanmay A. Wagh \\ Information Technology, \\ NBN Sinhgad School of \\ Engineering, \\ Pune, India
}

\author{
R. M. Samant \\ Assistant Professor \\ Department of \\ Information Technology, \\ NBN Sinhgad School of \\ Engineering, \\ Pune, India
}

\author{
Sharvil V. Gujarathi \\ Information Technology, \\ NBN Sinhgad School of \\ Engineering, \\ Pune, India
}

\author{
Snehal B. Gaikwad \\ Information Technology, \\ NBN Sinhgad School of \\ Engineering, \\ Pune, India
}

\begin{abstract}
Grapes (Vitis Vinifera) is basically a sub-tropical plant having excellent pulp content, rich color and is highly beneficial to health. Generally, it is very time-consuming and laborious for farmers of remote areas to identify grapes leaf diseases due to unavailability of experts. Though experts are available in some areas, disease detection is performed by naked eye which causes inappropriate recognition. An automated system can minimize these problems. The disease on the grape plant usually starts on the leaf and then moves onto the stem, root and the fruit. Once the disease reaches the fruit the whole plant gets destroyed. The approach is to detect the disease on the leaf itself in order to save the fruit. In our proposed system we have used a Deep Learning model named Convolutional Neural Network. Feature extraction and model training of the leaf images is performed using pre-defined AlexNet architecture. The image Dataset is taken from "National Research Centre for Grapes" (ICAR). It consists of images of diseases named Powdery mildew, Downy mildew, Rust, Bacterial Spots and Anthracnose. Image of the leaf is captured using the built-in camera module of a mobile phone. The accuracy achieved is $98.23 \%$ for powdery mildew vs bacterial spots.
\end{abstract}

\section{Keywords}

Deep Learning, Artificial Intelligence, Convolutional Neural Network, Alex-Net, Grapes leaf Disease

\section{INTRODUCTION}

Grapes are a popular fruit in India. Maharashtra is a leading state in production of grapes in India. Area under grapes in Maharashtra is about 86 thousand hectors and production is around 774 thousand tons of grapes annually [2]. While they are grown world-wide for making wines and raisins, they are mainly consumed as fresh fruits in India. Grapes also possess several health benefits due to their high nutrients content and can be used as natural remedies for several health problems. Due to their extensive use and benefits, and ease of growing in variable climatic conditions, cultivation of grapes has proved to be very profitable [1]. According to a report of 2011-12 from AgriXchange [2], India was the 18th largest, grapes producing country in the world, contributing to around $1,234.9$ thousand tons world's grapes production.

This report also presents an increasing trend of grapes production in the country in the years 2001-2011. This crop however is very susceptible to different diseases which have caused significant yield losses during this time. Though the disease severity varies from year to year, early and proper identification of diseases has proved to be a key factor for preventing high yield losses and maintaining quality of the crop through proper usage of pesticide. Proper diseaseidentification becomes difficult because of (i) similar symptoms of different diseases, (ii) different symptoms for a single disease at different stages of the crop, and (iii) presence of different diseases at the same time. So, a reliable and automatic method is required to avoid subjective errors in disease identification.

We have mainly focused on four diseases in grapes causing devastating yield losses in most of the years. These are Bacterial Spots, Powdery Mildew, Downy Mildew and Rust. The system will give more accurate results for the detection of these diseases using the CNN.

\section{LITERATURE SURVEY}

The automated method for the detection of disease affected on leaf is one of the important research areas as it provides many advantages in saving the fruit. Lots of research is carried out in this area but Artificial Intelligence is rarely seen being applied for this purpose.

In [3] Authors have proposed detection of diseases in the early stage by developing continuous monitoring system using various sensors like temperature, humidity, leaf wetness. ZigBee is used for wireless Data transmission between the farmer and the sensors. Hidden Markov Model is used to asses and to predict the disease at early stage.

In [4] multi-class SVM is used as a classifier and for feature extraction the RGB signal are converted into LGB form. In image pre-processing resizing, enhancing and smoothening of image is carried out to save the memory and reduce the processing time. For image Segmentation K- means clustering is carried out. Maximum $90 \%$ accuracy was obtained in testing the Black rot.

In [5] 120 images are taken directly from farms through mobile camera. Accuracy between different methods like SVM, BPN and fuzzy is compared and then SVM is used. In image preprocessing the image is resized to $226 \times 226$. RGB images are converted to HSV color space. Background subtraction is carried out to remove unwanted background in the image and to focus on leaf section. The average accuracy obtained is $89.3 \%$.

In [6] Authors have used $\mathrm{CNN}$ model to recognize and classify phenological stages of different types of plants based on the visual data i.e images which are captured every half an hour by the mounted cameras. Pre- trained Alex Net architecture is used in order to classify. The maximum 
accuracy obtained is $87.14 \%$ for Pepper which is more as compared to other traditional Machine Learning Approach.

In [7] a public dataset of 86,147 images of diseased and healthy plants are taken which consists of 57 different classes. In addition to the $\mathrm{CNN}$ architecture author have used generative Adverserial Networks where background segmentation is carried out and discriminator is used as a Classifier. Resnet was able to obtain $80 \%$ accuracy on the unsupervised dataset.

In [8] Authors have used 2 types of Flower dataset. Automated system is developed for the detection of the type of flower using CNN and Transfer Learning. Instead of building the model from scratch transfer learning provides easy access to pre-trained models like GoogleLeNet, OverFeat, Inception-v3, Xception etc. The trained model resides on the server side. User click the image which is converted to base 64 which is send to cloud storage. The converted image is send to $\mathrm{CNN}$ where the output class label is predicted. The proposed model achieved maximum accuracy of $98.66 \%$ for Inception-v3 model.

\section{CONVOLUTIONAL NEURAL NETWORK}

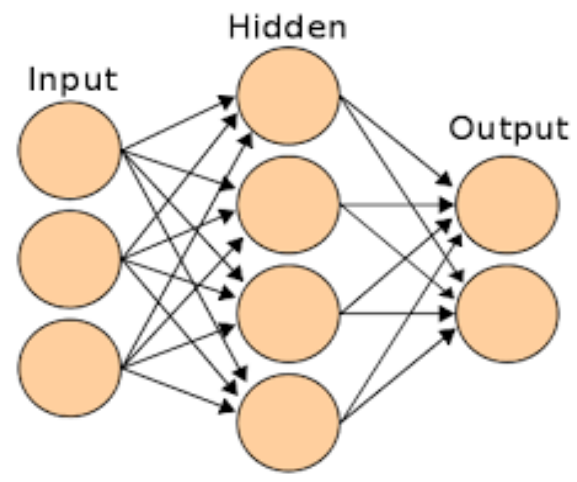

Figure 1.a

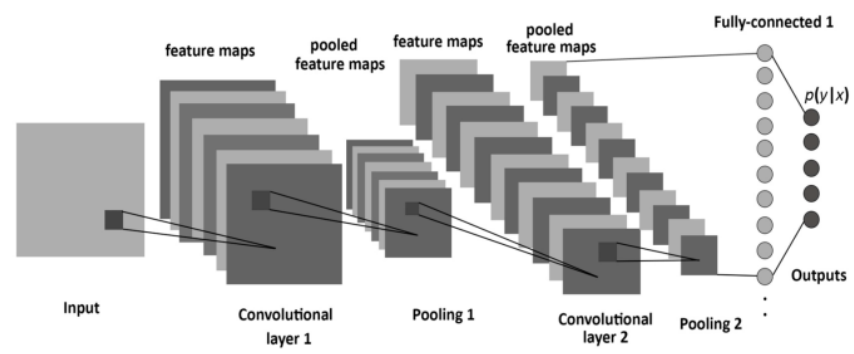

Figure 1.b

Artificial neural networks are motivated by the learning capabilities of the human brain which consists of neurons interconnected by synapses. ANNs are not suitable for images because these networks lead to over-fitting due to size of image. The major difference between a traditional Artificial Neural Network (ANN) and CNN is that only the last layer of a CNN is fully connected whereas in ANN, each neuron is connected to every other neuron as shown in Fig.2 Convolutional neural networks use images directly as input. Instead of handcrafted features, convolutional neural networks are used to automatically learn a hierarchy of features which can then be used for classification purposes. This is accomplished by successively convolving the input image with learned filters to build up a hierarchy of feature maps. The hierarchical approach allows learning more complex, as well as translation and distortion invariant, features in higher layers.

A Convolutional Neural Network (CNN) has four Type of layers as follows -

1. Convolutional Layer (CONV)

2. Rectified Linear Unit Layer (Re Lu)

3. Pooling Layer (POOL)

4. Fully-Connected Layer (FC)

\subsection{Convolutional Layer}

Convolution is the first layer which is used to extract features from an input image. It preserves the relationship between pixels by learning image features using small squares of input data. The Layer contains $\mathrm{N}$ filters which are small in size (for example [3×3] as in fig). These $3 \times 3$ filters are convoluted with the input image matrix by sliding the filter slide through the width and height of the image. Firstly, the feature matrix is multiplied pixel by pixel with the selected square from the image. Then the values are added and finally divided by the total number of pixels (in our example it is 9 due to $3 \times 3$ filter size). The obtained value is inserted in a new matrix. This process helps to reduce the image without loss of any feature.

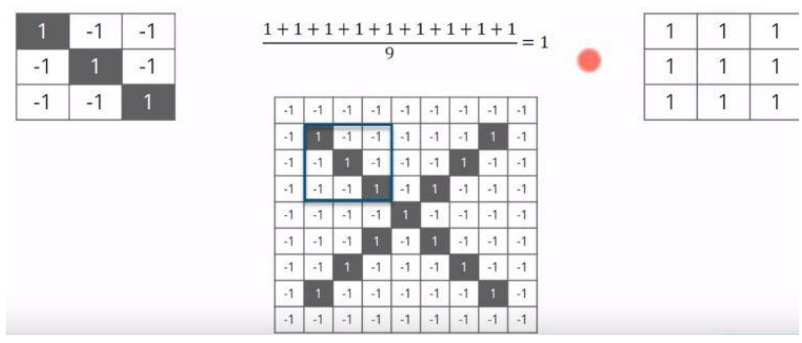

Figure 2

\subsection{Rectified Linear Unit Layer}

In $\mathrm{Re} \mathrm{Lu} \mathrm{layer} \mathrm{the} \mathrm{pixels} \mathrm{which} \mathrm{are} \mathrm{not} \mathrm{necessary} \mathrm{are}$ deactivated and only the important pixels are kept. From Conv layer we get positive as well as negative pixel values. The positive pixels are important for the further finding of features and the negative values are of less importance. The $\mathrm{Re} \mathrm{Lu}$ layer either converts the pixel to 0 or 1 . If the value of pixel is negative then it is converted to 0 and for any value greater than 0 it retains the same value.

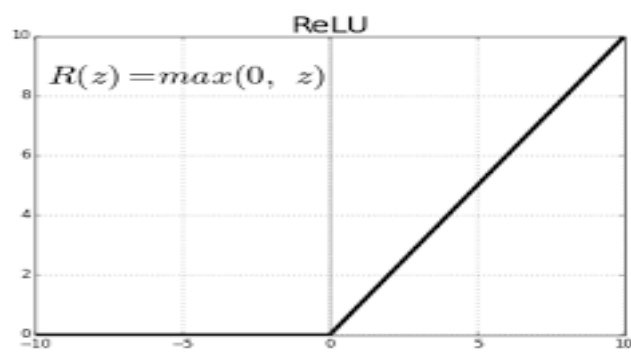

Figure 3

\subsection{Pooling Layer}

The Pooling layer does a simple job of down sampling or compressing the dimensions of the input image. A stride is selected which can be $2 \times 2$ or $5 \times 5$ etc. After the selection of stride it is applied to the dimension matrix obtained from the Conv Layer. Maximum value is taken from each stride and stored in a new matrix. Depending on the stride Pooling is of two types Max Pooling and Minimum Pooling. When the stride is large such Pooling is known as Max Pooling whereas 
small stride is known as Minimum Pooling. For example, if the input is [64*64*12] and if a stride of $2 \times 2$ is applied then after down sampling the output will be [32*32*12]. Figure 4 shows max Pooling Layer

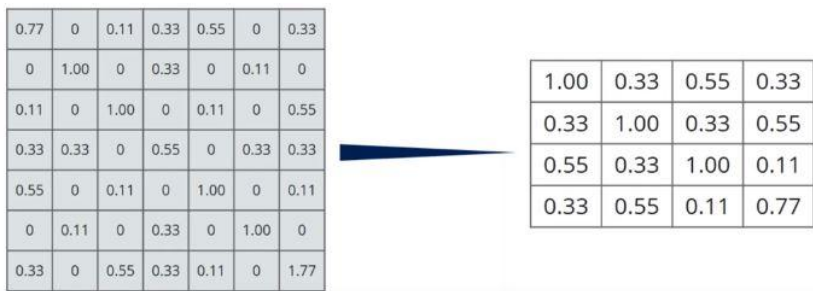

\subsection{Layer Stacking}

Figure 4

Multiple Layers are stacked of Convolution, $\mathrm{Re} \mathrm{Lu}$ and Pooling according to the architecture. The number of layers to be stacked varies as per use of the predefined architectures like Google Le Net, Alex Net etc. The output obtained is the minimized matrix of the input image.

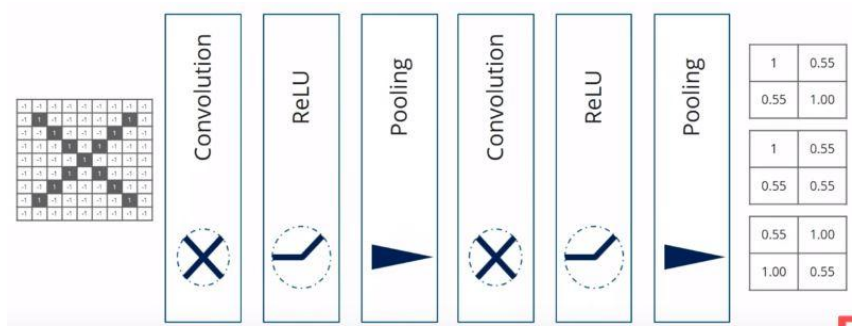

Figure 5

\subsection{Fully Connected Layer}

The Fully Connected Layer in CNN has neurons that are fully connected to all the neurons of the previous layer. Multiple FC layers are stacked as per the architecture used. It is often the last layer used in CNN which is responsible to predict the output or the label of the input class. Different activation functions are used like SOFTMAX which is used to classify multi-class problems. Hence, it has an output dimension of [1 $1 \mathrm{xM}]$ where $\mathrm{M}$ is the number of classes or labels used for classification.

\subsection{AlexNet Architecture}

In this paper we have used the AlexNet architecture [10] which strictly bounds us to use $227 \times 227 \times 3$ dimension. AlexNet consist of 5 convolution layers and 3 fully connected layers. The first two Convolutional layers are followed by the Overlapping Max Pooling layers. The third, fourth and fifth convolutional layers are connected directly. The fifth convolutional layer is followed by an Overlapping Max Pooling layer, the output of which goes into a series of two fully connected layers. The second fully connected layer feeds into a softmax classifier with 1000 class labels. Re LU nonlinearity is applied after all the convolution and fully connected layers.
Table 1. Architecture of pre-trained cnn, alexnet

\begin{tabular}{|c|c|c|}
\hline Layer & Sizes & Output $\div$ \\
\hline Inpur & - & $227 \times 227 \times 3$ \\
\hline Conv 1 & $11 \times 11 \times 3$ & $53 \times 55 \times 96$ \\
\hline Relu 1 & - & $55 \times 55 \times 96$ \\
\hline Norm 1 & - & $55 \times 55 \times 96$ \\
\hline Pooll & $2 \times 2 \times 96$ & $27 \times 27 \times 96$ \\
\hline Conv 2 & $3 \times 3 \times 48$ & $27 \times 27 \times 256$ \\
\hline Relu 2 & - & $27 \times 27 \times 256$ \\
\hline Norm 2 & - & $27 \times 27 \times 256$ \\
\hline Pool2 & $2 \times 2 \times 256$ & $13 \times 13 \times 256$ \\
\hline Couv 3 & $3 \times 3 \times 384$ & $13 \times 13 \times 384$ \\
\hline Relu 3 & - & $13 \times 13 \times 384$ \\
\hline Conv 4 & $3 \times 3 \times 192$ & $13 \times 13 \times 384$ \\
\hline Relu 4 & - & $13 \times 13 \times 384$ \\
\hline Conv 5 & $3 \times 3 \times 192$ & $13 \times 13 \times 256$ \\
\hline Relu 5 & $\cdot$ & $13 \times 13 \times 256$ \\
\hline Pool5 & $2 \times 2 \times 256$ & $6 \times 6 \times 256$ \\
\hline $\mathrm{FeIT}$ & $6 \times 6 \times 4096$ & $1 \times 1 \times 4096$ \\
\hline Reln 6 & - & $1 \times 1=4096$ \\
\hline Fc 2 & $1 \times 1 \times 4096$ & $1 \times 1 \times 4096$ \\
\hline Relu 7 & - & $1 \times 1=4096$ \\
\hline $\mathrm{Fe} 3$ & $1 \times 1 \times 4096$ & $1 \times 1 \times 1000$ \\
\hline Softurax & - & $1 \times 1 \times 1000$ \\
\hline
\end{tabular}

\section{PROPOSED SYSTEM}

\subsection{Dataset}

The Dataset is taken from Indian Council of Agriculture Research (ICAR) - National Research Grape Centre (NRGC) situated at Majri, Solapur Road India. Dataset consist of 1000 images each of Powdery Mildew, Bacterial Spots, Downy Mildew, Anthracnose, Rust and 1500 images of Healthy Leaves. Training set is chosen in such a way so that each class consist of same number of observation.

\subsection{Preprocessing}

The image dataset obtained consist of variable dimensions of images which are not accepted for the AlexNet framework. Hence, the images are broken into multiple images of dimensions $227 * 227$. Data Reduction is carried out where unnecessary images are removed. The unwanted data is to be removed to increase the performance after applying the model on the dataset.

\subsection{System Overview}

In figure 6 the proposed system overview is shown. The user simply captures the image of the grape leaf using a smartphone. User can also upload image from the gallery. Image can be cropped without processing further. Once, cropping is done the image is send to Node.js server through http Post method. The image path is saved on MySQL Database. Meanwhile, Acknowledgement is send to mobile by the Node.js server. Another server is already created in Python, on server side the trained CNN system on Grapes Leaf dataset is loaded which has 


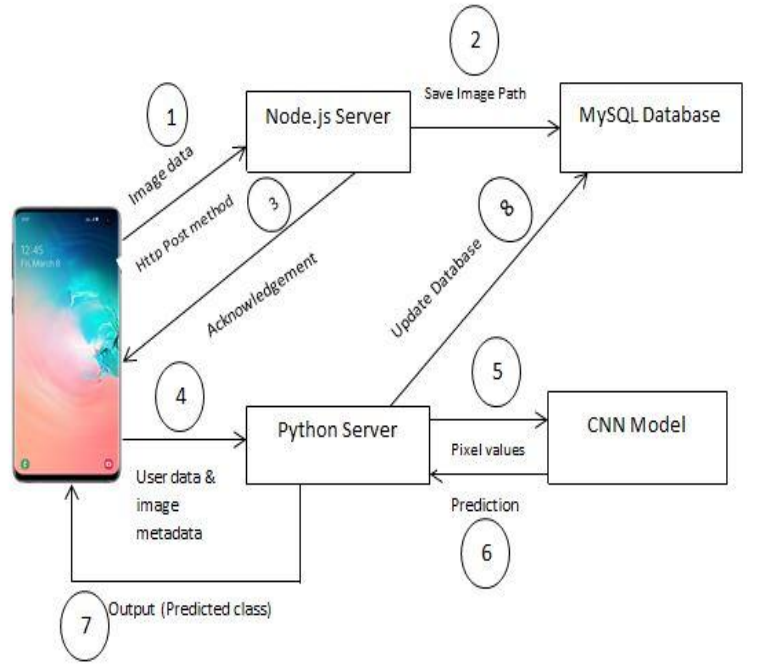

Figure 6

Extension as HDF5 file which contains the weights and biases of the pre-trained $\mathrm{CNN}$ model. Now, the server receives the User data and the image metadata. The pixel values of images are send to the pre-trained $\mathrm{CNN}$ model which returns the predicted output. Finally, Database is updated and the predicted output class are send to the mobile device.

\subsection{Software and Hardware Requirements}

$8 \mathrm{~Gb}$ Ram is used to train the CNN model. Two programming language are used in the proposed system. Python 3.6 is used to build the CNN model. Java is used for the android application development, to make user compatible to use the system. Efficient library of Python i.e 'Keras' is used to implement the AlexNet architecture. Other Python libraries like Numpy, h5py, sciPy are used. After training the model the resulting feature matrix is stored locally in .hdf5 format which is of $700 \mathrm{mb}$.

\section{MATHEMATICAL MODEL}

Let $\mathrm{S}$ be Closed system defined as,

$\mathrm{S}=\{\mathrm{Ip}, \mathrm{Op}, \mathrm{Ss}, \mathrm{Su}, \mathrm{Fi}, \mathrm{A}\}$

Training a Convolutional Neural Network using images of grapes disease leaf and performing various actions from the set of actions A so that Su state can be attained.

$\mathrm{S}=\{\mathrm{Ip}, \mathrm{Op}, \mathrm{Ss}, \mathrm{Su}, \mathrm{Fi}, \mathrm{A}\}$

Where,

$\operatorname{Ip} 1=\{\mathrm{Dtr} \mid \mathrm{Dtr} € \mathrm{D}\}$

Where,

Dtr - Images of an affected leaf

D - Dataset containing Images of grapes leaf

Output set :

$\mathrm{Op} 1=\{\mathrm{F} \mid \mathrm{F}$ is Acceptance of relevant Data $\}$

$\mathrm{Op} 2=\left\{\mathrm{F}^{\prime} \mid \mathrm{F}^{\prime}\right.$ is a set of features selected for extraction $\}$

$\mathrm{Op} 3=\left\{\mathrm{F}^{\prime}, \mid \mathrm{F}^{\prime}\right.$ ' is a set of features learned by the CNN through Training $\}$

$\mathrm{Op} 4=\{\mathrm{c} \mid \mathrm{c}$ is a trained model used to make predictions $\}$

Set of actions $=\mathrm{A}=\{\mathrm{F} 1, \mathrm{~F} 2, \mathrm{~F} 3, \mathrm{~F} 4\}$
Where,

$$
\begin{aligned}
& \text { F1 = Accepting Relevant Data } \\
& \text { F2 = Using Convolution Layer to extract features } \\
& \text { F3 = Training the Deep Neural Network } \\
& \text { F4 = Model Architecture Successful }
\end{aligned}
$$

Ss- Loading Images $\rightarrow$ Extracting Features $\rightarrow$ Training Model $\rightarrow$ Making Predictions

Su- Success state is when the CNN can accurately detect and classify the type of Disease.

Fi- Failure state is when the trained model makes an incorrect prediction.

\section{RESULTS}

The overall Grapes Leaf Disease Detection problem is divided into two parts. Firstly, the features from the images in the training dataset are extracted using the $\mathrm{CNN}$ network and is saved in a HDF5 format. Secondly, random test images are given to the network through android application. Two diseases at a time are tested. The maximum accuracy of 98.23\% is obtained from Bacterial Spots vs Powdery Mildew where 1000 images of each disease were taken to train. Table shows all the results obtained from this model.

\begin{tabular}{|c|c|}
\hline Diseases & Accuracy \\
\hline Bacterial Spots VS Rust & $62.14 \%$ \\
\hline $\begin{array}{c}\text { Downey Mildew VS } \\
\text { Powdery Mildew }\end{array}$ & $78.26 \%$ \\
\hline Rust VS Powdery Mildew & $89.67 \%$ \\
\hline $\begin{array}{c}\text { Bacterial Spots VS Powdery } \\
\text { Mildew }\end{array}$ & $98.23 \%$ \\
\hline
\end{tabular}

\section{CONCLUSION}

The objective of this paper is to predict the type of Disease on the image of grape leaf. To predict this Convolutional Neural network is used which is an unsupervised method. Alex-Net Architecture is used to train the model. It is observed from the results that Bacterial Spots Vs Powdery Mildew achieves maximum accuracy of $98.23 \%$.

\section{ACKNOWLEDGMENTS}

We greatly acknowledge Dr. Sawant and the Indian Council of Agriculture Research (ICAR) - National Research Grapes Centre (NRGC) for providing us information related to the diseases and most importantly giving accesses to the Image Dataset of the grapes Leaf.

\section{REFERENCES}

[1] A report of the expert consultation on viticulture in Asia and the Pacific May 2000, Bankok, Thailand. RAP publication:2000/13.

[2] http://agriexchange.apeda.gov.in/Market\%20Profile/one/ GRAPES.aspx

[3] Suyash S. Patil, Sandeep A. Thorat. "Early Detection of Grapes Diseases Using Machine Learning and IOT", 2016 Second International Conference on Cognitive Computing and Information Processing (CCIP)

[4] Nitesh Agrawal, Jyoti Singhai, Dheeraj K. Agrawal, "Grape Leaf Disease Detection and classification Using 
Multi-class Support Vector Machine", Proceeding International conference on Recent Innovations is Signal Processing and Embedded Systems (RISE-2017) 27-29 October,2017.

[5] Harshal Waghmare, Radha Kokare, " Detection and Classification of Diseases of Grape Plant Using Opposite Colour Local Binary Pattern Feature and Machine Learning for Automated Decision Support System”, 2016 3rd International Conference on Signal Processing and Integrated Networks (SPIN)

[6] Hulya Yalcin, "Plant Phenology Recognition using Deep Learning : Deep-Pheno".
[7] Emanuel Cortes, "Plant Disease Classification Using Convolutional Networks and Generative Adversial Networks".

[8] I.Gogul, V.Sathiesh Kumar, "Flower Species Recognition System using Convolutional Neural Networks and Transfer Learning", 2017 4th International Conference on Signal Processing, Communications and Networking (ICSCN -2017), March 16 - 18, 2017, Chennai, INDIA.

[9] Alex Krizhevsky, Ilya Sutskever, Geoffrey E. HInton ' Image Net Classification with Deep Convolutional Neural Networks. 